\title{
Development of Optical Fiber Bragg Grating Sensors for Structural Health Monitoring
}

\author{
Yukihiro SHIMADA and Akihiko NISHIMURA \\ Applied Laser Technology Institute, Tsuruga Head Office, Japan Atomic Energy Agency \\ 65-20 Kizaki, Tsuruga, Fukui, 914-8585, Japan \\ E-mail: shimada.yukihiro@jaea.go.jp
}

\begin{abstract}
A fiber Bragg grating (FBG) sensor with heat resistance was produced via a point microfabrication technique using a femtosecond pulse laser. The FBG sensor was reinforced by weaving with a carbon fiber tape. As a result, both high strength and a structure for mounting the sensor were readily incorporated. In a high-temperature tolerance evaluation of the FBG sensor, the temperature and strain were measured by the sensor at $600^{\circ} \mathrm{C}$. Therefore, the sensor is effective, and in addition, the problems associated with mounting the sensor on high-temperature piping have been eliminated. DOI:10.2961/jlmn.2013.01.0021
\end{abstract}

Keywords: atomic power plants, seismic safety, high-temperature environment, fiber Bragg grating, point-by-point method, carbon fiber

\section{Introduction}

It is very important to monitor the seismic deformation of aging piping systems in atomic power plants or chemical plants. For this purpose, many investigations of fiber sensors have been pursued $[1,2]$. Among them, the conventional fiber Bragg grating (FBG) sensor is the most promising for practical use. For example, a prototype FBGmonitoring system was installed at JOYO, the fast neutron material research reactor of the Japan Atomic Energy Association (JAEA). To the best of our knowledge, it was the first demonstration of the successful monitoring of the thermal expansion of the coolant pipes of the primary loop of the fast neutron reactor [3].

However, in that demonstration, a heat insulator was necessary to protect the FBG sensor from thermal damage. Therefore, the monitoring system could not effectively detect seismic vibration. Measurements were only carried out for thermal expansion [3]. To monitor the real-time vibration of the coolant pipe, it is necessary to directly bond the heat-resistant FBG sensor onto the surface of the hightemperature coolant pipes without a heat insulator. Thus, we developed a prototype FBG sensor using a femtosecond laser by point-by-point (PBP) microscopic processing [4]. For example, this successful approach could lead to new monitoring systems for use on the coolant pipes of a helium-gas-cooled-high-temperature reactor. In this report, we present further technical improvements on the new FBG sensors to make them suitable for application in atomic power plants. Two issues needed to be addressed: how to reinforce the fragile FBG sensor, which was accomplished with a carbon fiber; and how to test the reinforced FBG sensor at $600^{\circ} \mathrm{C}$ under loading conditions. In this case, the wavelength shift of the reflection was measured. A tunable fiber laser was also tested for the real-time monitoring of the FBG reflection.
2. Microfabrication of the fiber Bragg grating by the PBP method using a femtosecond pulse laser

The processing system for the fabrication of the FBG is composed of the laser system, the optics system of the microscope, and the optical fiber movement stage. The FBG sensor produced by the PBP method has excellent heat resistance and a degree of freedom as design characteristics $[5,6]$.

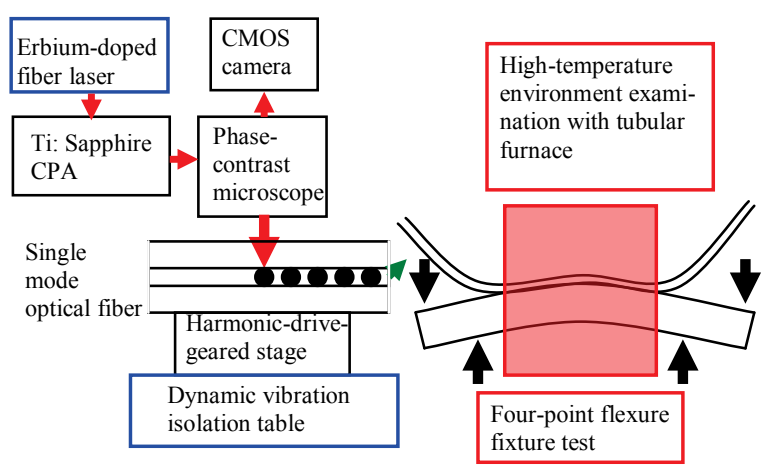

Fig. 1 Schematic of the processing system and the hightemperature tolerance examination of the FBG sensor

\subsection{Laser system for the microfabrication of the FBG}

A CPA pulse laser (Thales, model Alpha-10) that can generate a 100 -fs pulse duration with a $10-\mathrm{mJ}$ pulse energy at a $10-\mathrm{Hz}$ repetition was used for the FBG fabrication (Fig 1). This laser system has a femtosecond fiber oscillator (Menlo Systems, model T-Light 780), a regenerative amplifier and a 4-path amplifier. However, because high-energy laser light damages the object lens of this microscope, the entering light is limited to lesser or equal to $100 \mu \mathrm{J}$. Therefore, the 4-path amplifier is not working when the laser light passes through it. At the output of the regenerative 
amplifier, an air-gap polarizer and a 1/2 lambda wave plate are used to attenuate the laser pulse energy. As a result, the output laser energy can be controlled to as low as lesser to or equal to $1 \mu \mathrm{J}$. With respect to the moving speed of the sample stage, this processing system is changeable according to the repetition cycle of the laser. Therefore, the stability of the frequency of the main oscillator directly influences the processing accuracy. The repetition frequency of the laser system is $100 \mathrm{MHz}$, and this value is one digit higher or more stable than the titan sapphire laser.

\subsection{Phase-contrast microscopy for laser processing}

The processed laser light passes into the microscope (Olympus, model BX41) and then follows the same axis as the observation light. The processing is done from the side of the optical fiber through the object lens. Because the optical fiber has a cylindrical geometry, the focus depth position is different for the long and short axes. The cylindrical geometry is canceled in optics by filling the space surrounding the optical fiber with an index-matching liquid ( $\mathrm{n}=1.52,1$-bromonaphthalene) to correct this difference and make the laser incident to the side plane with the cover glass. The machining state is always observed with a CMOS camera set up in the microscope. Moreover, a phasecontrast microscope is used to identify the core and cladding of the optical fiber, even if the refractive index difference is small. In the microfabrication of a transparent medium, the shape of the processing point is greatly influenced by the numerical aperture (NA). The focal length of the object lens (Olympus, model PLN40XPH) used in this processing system is $4.5 \mathrm{~mm}$, and the NA is 0.65 . Because the beam diameter of the laser used for the processing is approximately $5 \mathrm{~mm}$, the effect of the NA is estimated to be approximately 0.55 . The energy of the laser pulse during irradiation was approximately $5 \mu \mathrm{J}$.

\subsection{Fixation and movement of the sample for pro- cessing}

The optical fiber was fixed on a slide glass plate with an index-matching oil and processed while moving slightly on a closed-loop-controlled stage using a harmonic gear drive (Sigma tech, model FS-1040SPX(MD)) and a 3-axis piezo drive stage (Sigma koki, model FINE-503). Because of the decreased vibration, the stage moves at a fixed velocity without stopping. The moving speed determines the processing interval for the FBG, because the period of exposure time for the pulse laser is always constant. This method has the advantage of suppressing any decrease in accuracy originating from the stopping and starting of the sample moving stage. The sample stage is controlled by LabVIEW software (National Instruments). The microscope for processing and the sample moving stage are set up on a dynamic vibration isolation table (JRS Scientific Instruments, model AVI-150). This table suppresses the vibration by detecting it and moving in the opposite phase. Vibrations with a frequency of lesser or equal to $200 \mathrm{~Hz}$ are reduced by the active suppression vibration function, and any additional vibration is absorbed by the rubber insulator. Because this table removes not only the vibration of the room but also that generated by the process, it is particularly advantageous in microfabrication.

\subsection{Optical properties of the produced FBG sensor}

The Bragg reflection wavelength can be described as a function of the period of the refractive index.

$$
\lambda=2 \mathrm{n} \Lambda / \mathrm{m},
$$

where $\Lambda$ is the modulation period of the refractive index of the fiber core, $\mathrm{n}$ is the average of the refractive index, and the integer $\mathrm{m}$ is the reflection order. The period of the irradiation was $1626 \mathrm{~nm}$, and 800 points were processed in the optical fiber core. Thus, the resulting Bragg reflection wavelength of the FBG is approximately $4710 \mathrm{~nm}$. In addition, the light is reflected in multiples of the frequency, and thus, the third-order Bragg reflection wavelength becomes $1570 \mathrm{~nm}$. A spectrum analyzer (Yokogawa, model AQ6370B) and ASE broadband light source (Amonics, model ALS-18-B) were used to measured the characteristic reflection spectrum of the fabricated FBG sensor. The spectrum of a white light source normalizes the wavelength spectrum data from the FBG. The central wavelength of the reflected spectrum of the FBG was $1569.2 \mathrm{~nm}$, and the full width at half maximum was $1.3 \mathrm{~nm}$ (Fig. 2).

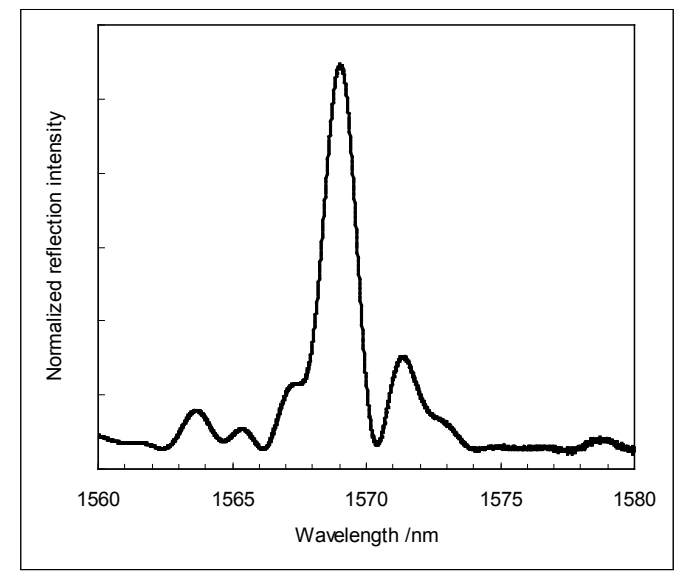

Fig. 2 Reflection wavelength spectrum of the fabricated FBG. The interval of the irradiation was 1626 $\mathrm{nm}$, and 800 points were processed.

\section{Performance assessment of the produced FBG sen-} sors

Generally, the performance of a sensor and the installation and operation method are important in determining its measurement accuracy. In particular, the mounting system is an important issue because FBG sensors are fundamentally contact-type measurement devices. Therefore, the FBG sensor was reinforced with woven carbon fibers. Then, the heat tolerance of the sensor was evaluated when it was bonded onto a steel sample using a ceramic adhesive.

\subsection{Reinforcement of the FBG sensor with a carbon fiber}

Because an optical fiber is fragile and delicate, it is difficult to handle. To address this problem, the optical fiber was woven with a carbon fiber fabric tape. The carbon fi- 
ber fabric tape was $15 \mathrm{~mm}$ in width and $70 \mathrm{~mm}$ in length. It was formed in a manner such that a weft yarn was woven in a direction substantially perpendicular to the warp yarn, and wherein an optical fiber was included in the center warp. Femtosecond-laser processing was used to fabricate 17 FBGs in the optical fiber. Figure 3 shows the terminal of the carbon fiber fabric tape. The master-reinforced carbon tape with the FBGs was cut into four pieces for use in the following experiments.

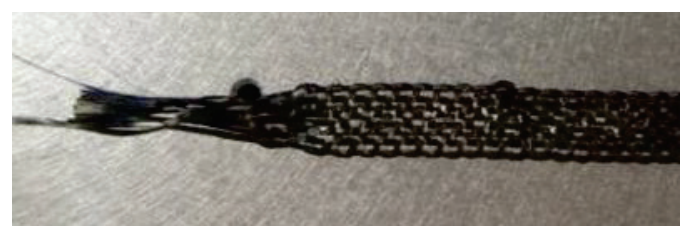

Fig. 3 Prototype -reinforced sensor with an optical fiber containing the FBGs woven with a carbon fiber fabric tape.

\subsection{Sample for measurement and adhesion}

The field test of the sensor measured the strain and the temperature after bonding it to STPA24 steel. This STPA24 chromium-molybdenum steel is a material used for the second system heat transfer piping of a sodium fast reactor. Two types of adhesives (Cotronics, model Resbond 954 and 940) with different coefficients of thermal expansion (CTE) were investigated. The CTEs of the steel and the adhesives are shown in Table 1.

Table 1 CTEs of the sample and the adhesive

\begin{tabular}{cl}
\hline Material & $\mathrm{CTE} /{ }^{\circ} \mathrm{C}^{-1}$ \\
\hline STPA24 & $12-14 \times 10^{-6}$ \\
Resbond 954 & $18.0 \times 10^{-6}$ \\
Resbond 940 & $8.10 \times 10^{-6}$ \\
\hline
\end{tabular}

\subsection{Comparison of measurements with an electrical- conductor-type strain gauge and the FBG sensor}

First, the FBG sensor was calibrated using a strain gauge sensor at room temperature. For this purpose, the correlation of the FBG sensor and the strain gauge sensor was measured by applying a load to the sample. The fourpoint flexure fixture test evaluated the correlation between the strain and the fabricated FBG sensor, as shown in Fig. 4.

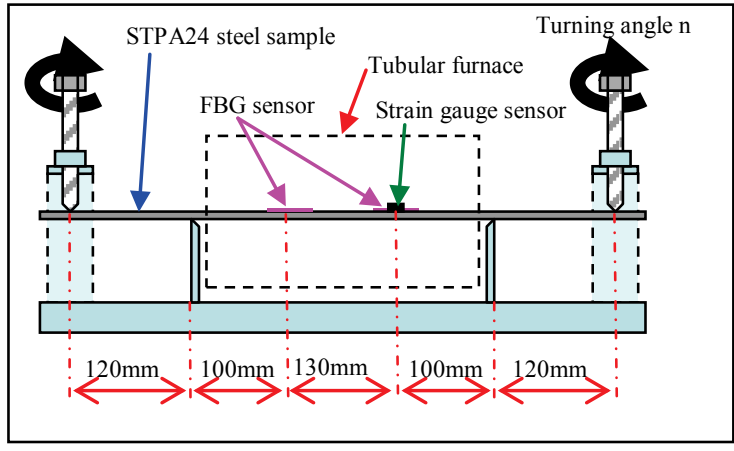

Fig. 4 Schematic of the four-point flexure fixture measurement. The relationship between the turning angle and the occurring strain is examined using a strain gauge sensor at room temperature.

This experiment measured the correlation of the load device transformation and the strain of the material. Additionally, the laser displacement sensor (Keyence, model LC-2400) measured the external amount of the transformation without any contact, and the strain of the material was presumed indirectly. Based on the results of this test, it was determined that the FBG sensor was able to detect within 300 microstrains $(\mu \varepsilon)$.

\subsection{Strain measurement examination at high tempera- ture}

A high-temperature tubular furnace (Asahi-rika, model ARF-30KC) was used to heat the sample when there was no load. Precise temperature control was achieved with a controller (Omron, model E5AR). The rate of the temperature increase was $240^{\circ} \mathrm{C}$ per $\mathrm{h}$ in this experiment. Figure 5 shows the temperature dependence of the Bragg wavelength without a load. The reflection wavelength from the FBG shifts linearly from room temperature to $600^{\circ} \mathrm{C}$. The coefficient of the temperature change and wavelength shift is $13 \mathrm{pm} \cdot{ }^{\circ} \mathrm{C}^{-1}$. There was a slight delay from the time the temperature changed, and a change in the Bragg wavelength was detected. This delay of the thermal conduction is caused by the presence of the adhesive between the object and the FBG sensor.

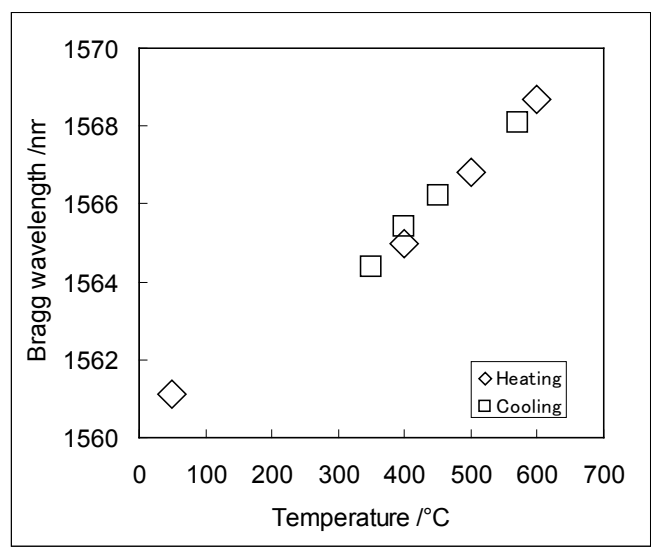

Fig. 5 The Bragg wavelength of an FBG sensor as a function of temperature. 
Then, the strain was determined at different temperatures after stabilization for 10 minutes. Initially, the correlation between the strain gauge sensor and the fabricated FBG sensor was measured at room temperature. However, the strain gauge sensor cannot be used at high temperature because it would be damaged. Therefore, the amount of the strain shown on the horizontal axis of Fig. 6 has been converted from the turning angle of the four-point flexure fixture measurement machine.

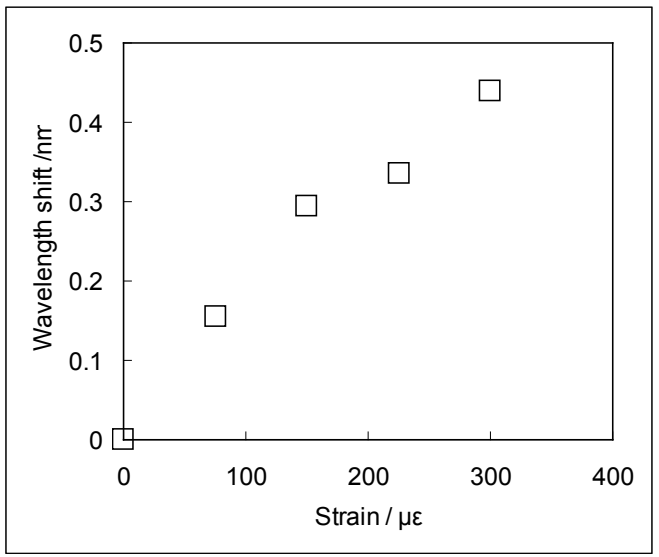

Fig. 6 Shift in the Bragg wavelength of an FBG as a function of strain at $400^{\circ} \mathrm{C}$. The coefficient of the strain and wavelength shift is $1.5 \mathrm{pm} \cdot \mu \varepsilon^{-1}$.

\section{Discussion}

The results shown here are not sufficient to enable the practical use of the FBG sensor system in an actual plant. The outline for an FBG-monitoring system for the inspection of nuclear power plants has been described. Straight and elbow parts are used to construct the heat piping in a nuclear power plant. It is important to measure the transformation in the part of the elbow that undergoes thermal expansion. Figure 7(a) shows a downsized elbow-pipe model of the coolant piping system. Multipoint sensing with FBGs sensors having different reflected wavelengths located at one of the pipe elbows could be possible. These FBGs would be fixed around the jointed circular line of the pipe elbow and the straight pipe, as in Fig. 7(b).

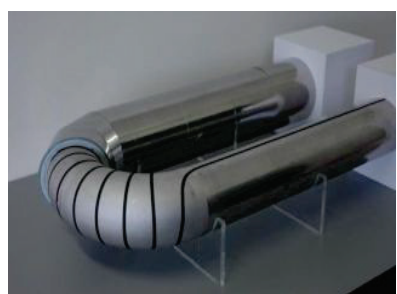

(a)

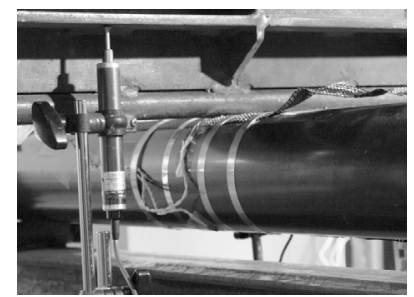

(b)
Fig. 7 Examination of a carbon-reinforced FBG sensor

(a) Downsized-elbow-pipe model

(b) Carbon-reinforced sensor on the loading machine

The fabrication method shown in Fig. 1 can produce a sensor with an arbitrary wavelength. However, it should be improved to increase the productivity for multipoint FBG sensors. Thus, it is necessary to examine the following is- sues to use the prototype-reinforced FBG sensor in atomic power plants.

First, it is necessary to increase the repetition rate of the processing laser to increase the productivity of the process for manufacturing the FBG structure. Upgrading the pumping laser of the present CPA laser system could increase its repetition rate. Specifically, LD-pumped green pulse lasers may be practical. Moreover, all-in-one shoe-box-sized lasers may become commercially available due to the advanced cooling performance of LD direct-pumping ytterbium-doped gain materials [7, 8]. A CPA amplifier based on an extended mode area is capable of generating laser pulses with a repetition rate of $100 \mathrm{kHz}$, a laser pulse duration of 1 ps, and a laser pulse energy of $10 \mu \mathrm{J}$.

Second, as with any contact-type sensors, with an FBG sensor, it is important for the measurement object and the sensor to be in close contact for the accurate performance of the sensor. However, it is difficult to maintain adhesive strength over the wide temperature range from room temperature to high temperatures $\left(600^{\circ} \mathrm{C}\right)$. Adhesive strength decreases when the coefficients of thermal expansion of the object and the adhesive are different. It is impossible to equate the coefficients of the thermal expansion of the object being measured, the sensor, and the adhesive. Therefore, it is ideal to directly connect the material to the sensor without the use of an adhesive. Laser micro welding technology for different types of material will become an important method for addressing this issue [9].

Finally, in bonding, it is very important during preparation to make sure that both sides of the objects to be bonded are clean and smooth. From a practical viewpoint, it must be expected that the material surface of the piping in a plant will be dirty, and thus have a negative impact on the strength of the bond to the sensor when it is mounted. Therefore, the use of laser ablation, an extremely effective method for rapidly cleaning surfaces, should be considered [10]. Infrared pulse lasers could also be used to remove rust layers and radioactive scale from structural materials [11] For the maintenance of atomic power plants, it is anticipated that a specially designed robot with a compact highrepetition pulse laser and various sensors for determining the working distance will be used for such cleaning of pipe surfaces.

\section{Conclusion}

Ultrashort laser micro processing was used to produce a heat-resistant FBG sensor by a point-by-point method. This FBG sensor successfully measured changes in the temperature and strain at temperatures up to $600^{\circ} \mathrm{C}$. A ceramic adhesive was effective for strongly bonding the FBG sensor to the sample pipe at temperatures up to $600^{\circ} \mathrm{C}$. However, it is necessary to select the bonding material carefully. It is difficult to match it accurately though it is ideal that the bonding object is equal to CTE of adhesive. The adhesive of a CTE that was larger than the bonding object maintained adhesive power in this experiment. Like generalresistance-line-type strain sensor, measurements obtained with the FBG sensor contain both temperature change and strain data. However, the two measurements can be separated by changing the mounting method. If only the one side of the sensor is bonded, measurements of the temperature can be obtained. This is a general distortion measure- 
ment method that can be used also with the resistance line type sensor. The possibility that this new type of FBG sensor could be effective in the tube of a heat exchanger in a fast reactor was verified on the basis of the results of these experiments.

\section{Acknowledgments}

We deeply appreciate Mr. H. Suzuki and Mr. H. Ishibashi of Kumagai Gumi Co., Ltd for their assistance with the heating and adhesion tests. We also appreciate Mr. H. Kawahara and Dr. K. Saruta of JAEA for their help with the heating test and preparation of the samples. We also thank Dr. C. Ito and Dr. T. Aoyama for mounting the FBG sensor on the coolant pipe of the FBR.

\section{References}

[1] A. Othonos: Rev. Sci. Instrum., 68, (1997) 4309.

[2] K. O. Hill, Y. Fujii, D. C. Johnson, and B. S. Kawasaki: Appl. Phys. Lett., 32, (1978) 647.
[3] K. Matsuba, C. Ito, H. Kawahara and T. Aoyama: Journal of Power and Energy Systems, 2, (2008) 545.

[4] Y. Shimada, A. Nishimura, M. Yoshikawa and T. Kobayashi: J. Laser Micro/Nanoeng., 5, (2010) 99.

[5] Y. Kondo, K. Nouchi, T. Mitsuyu, M. Watanabe, P.G. Kazansky and K. Hirao: Opt. Lett., 24, (1999) 646.

[6] A. Martinez, M. Dubov, I. Khrushchev, I. Bennion: Electron. Lett., 40, (2004) 1170.

[7] Y. Zaouter, J. Boullet and E. Cormier: Opt. Lett., 33, (2008) 1527.

[8] M. Larionov, F. Butze, D. Nickel and A. Giesen: Opt. Lett., 3, (2007) 494.

[9] Y. Ozeki, T. Inoue, T. Tamaki, H. Yamaguchi, S. Onda, W. Watanabe, T. Sano, S. Nishiuchi, A. Hirose and K. Itoh: Appl. Phys. Express, 1, (2008) 08260.

[10] W. Zapka, W. Ziemlich, and A. C. Tam: Appl. Phys. Lett., 58, (1991) 2217.

[11] C. Konagai, Y. Sano, K. Nittoh and A. Kuwako: IEEJ Transactions on Electronics, Information and Systems, 123, (2003) 198. (in Japanese)

(Received: June 08, 2011, Accepted: January 05, 2013) 